\title{
The Effect of Omission of the Bladder Flap Formation at Lower Segment Cesarean Delivery: A Randomized Controlled Trial
}

\author{
Yasmeen A. Youssef, Tarek A. Farghaly, Elwany Elsenosy, Ahmed A. Youssef, \\ Ahmed M. Abbas* \\ Department of Obstetrics \& Gynecology, Faculty of Medicine, Assiut University, Assiut, Egypt \\ Email: *bmr90@hotmail.com
}

How to cite this paper: Youssef, Y.A., Farghaly, T.A., Elsenosy, E., Youssef, A.A. and Abbas, A.M. (2019) The Effect of Omission of the Bladder Flap Formation at Lower Segment Cesarean Delivery: A Randomized Controlled Trial. Open Journal of Obstetrics and Gynecology, 9, 1083-1091. https://doi.org/10.4236/ojog.2019.98105

Received: July 6, 2019

Accepted: August 3, 2019

Published: August 6, 2019

Copyright $\odot 2019$ by author(s) and Scientific Research Publishing Inc. This work is licensed under the Creative Commons Attribution International License (CC BY 4.0).

http://creativecommons.org/licenses/by/4.0/

\begin{abstract}
Background: This study aims to evaluate the effects of omission of the bladder flap formation at primary and repeat lower segment CS. Patients and Methods: The current study was randomized controlled trial conducted in Women Health Hospital, Assiut University, Egypt between March 2017 and May 2018 (ClinicalTrial.gov NCT03016273). Patients were divided into: Non bladder flap group: Uterine incision made $1 \mathrm{~cm}$ above the vesico-uterine reflection without incision and dissection of the bladder peritoneum and bladder flap group: Standard cesarean section technique with incision and dissection of a bladder flap prior to uterine incision. Results: The study included 150 patients (75 in each arm). The most common indication for CS in both groups was repeated CS. Non-bladder flap group, compared with flap group, showed shorter skin-incision to delivery time and total operative time, and significantly lower mean estimated blood loss and postoperative pain score. Non-bladder flap group, compared with flap group, was more likely to show postoperative microhematuria. The two groups required approximately the same time for post-operative defecation. Conclusion: Omission of bladder flap formation during CS is associated with shorter operative time, less blood loss, less postoperative pain and lower incidence of postoperative hematuria.
\end{abstract}

\section{Keywords}

Cesarean Section, Bladder Flap, Hematuria, Lower Segment

\section{Introduction}

Cesarean section (CS) rates increased from 6.7\% in 1990 to $19.1 \%$ in 2014, which 
represents a $12.4 \%$ absolute increase. Less developed countries showed the largest absolute increase [1] [2]. At country level, Egypt, Tunisia and Morocco witnessed the largest rise in the African region. CS rates in Egypt rose from $4.6 \%$ to $51.8 \%$ over the 24-year period. Along with Morocco, these two countries had the largest worldwide rise in CS rates (11.6\%) [3].

With the increasing numbers of CSs, there is the need to utilize evidence-based techniques to optimize outcomes and minimize complications. Recently, the case has been made for simplifying the procedure by eliminating unnecessary and potentially harmful steps [4]. Creation of the bladder flap is an integral step of the standard CS. The bladder flap is made by superficially incising and dissecting the peritoneal lining to separate the urinary bladder from the lower uterine segment. Started in the pre-antibiotic era, the idea for the bladder flap was to enable the surgeon to gain access to the lower uterine segment while minimizing injury to the bladder. Its subsequent closure was supposed to protect the peritoneal cavity from intrauterine infection. Since then, closure of the bladder flap has been demonstrated to be unnecessary and has been abandoned. The bladder flap however, continues to be performed without evidence of benefit. Evidence on the role of the bladder flap in cesarean section is very limited [5] [6].

In emergent CS where rapid delivery is the goal, the bladder flap step is commonly removed. A simplified method of CS (Pelosi-type) including cancellation of the bladder dissection among other modifications has been shown to be safe and cost saving. The paucity of evidence for or against this commonly utilised technique in CS is the rationale for this study [4].

This study aims to evaluate the effects of omission of the bladder flap formation at primary and repeat lower segment CS.

\section{Patients and Methods}

The current study was randomized controlled trial conducted in Women Health Hospital, Assiut University, Egypt between March 2017 and May 2018. This trial was recorded on clinical trials with number ClinicalTrial.gov NCT03016273. The institutional ethical review board approved the study protocol. An informed written consent has been obtained from those patients who will participate in the study mentioning the risk of the procedure and associated anesthesia.

\subsection{Sample Size}

Sample size was calculated using $G^{*}$ power, version 3.1.9.2. Based on previous study the mean of incision to delivery time for Bladder flap was $23 \pm 19.6$ and for No bladder flap was $15.5 \pm 121$. With a power of $80 \%$ (using two sided test and a of 0.5 ) the sample needed for the study was estimated to be about 150 patients (75 in each group).

\subsection{Inclusion Criteria}

1) Patients undergoing primary or repeat CS. 
2) Women pregnant at gestational age $>32$ weeks.

3) Age 18 - 45 years.

\subsection{Exclusion Criteria}

1) Patients undergoing planned vertical uterine incision.

2) Patients underwent previous laparotomies.

3) Gestational age less than 32 weeks.

4) Patient refusing to participate in the study.

\subsection{Allocation and Concealment}

All patients who presented to Women's Health Hospital and meet the listed inclusion and exclusion criteria were invited to participate in the study.

Patients were divided into:

- Non bladder flap group: Uterine incision made $1 \mathrm{~cm}$ above the vesico-uterine reflection without incision and dissection of the bladder peritoneum.

- Bladder flap group: Standard CS technique with incision and dissection of a bladder flap prior to uterine incision.

\subsection{Randomization}

The women were randomly allocated to bladder flap group or non bladder flap using computer generated random number tables and opaque sealed envelopes containing the participants' group allocation. The envelopes were prepared and sent to an assigned nurse, who opens each envelope just before CS. All patients were blinded to the allocation to avoid bias.

\subsection{Intervention}

\section{The following data were obtained for every case:}

a) Demographic data: Age, weight in $\mathrm{Kg}$, height in $\mathrm{cm}$, residence, educational level, Parity, number of CS, gestational age in weeks and Indication of CS.

Fetal presentation

b) Intervention variables: Operative time, incision time, delivery time, skin closure time, and any intraoperative complications.

c) Post-intervention variables: Morbidity and mortality, postoperative pain score, need for blood transfusion, bladder injury, and finally urine sample was collected directly through the Foley catheter before its removal to be tested with dipsticks for microhematuria.

\subsection{Surgical Technique}

Cesarean deliveries were performed by well-trained senior residents supervised by assistant lecturer or obstetrician staff. Preoperatively urine sample was collected directly through the Foley catheter and tested with dipsticks for microhematuria.

CS was performed under spinal anesthesia, using a 25-gauge needle to inject 
$0.5 \mathrm{mg}$ morphine and $12 \mathrm{mg} 0.5 \%$ bupivacaine. No vasoconstrictor was used unless the blood pressure dropped by $20 \%$ from the baseline value; if this was the case, $10 \mathrm{mg}$ ephedrine was given.

CS technique was the same in all recruited women except at the bladder flap step. A Pfannenstiel incision 2 to $3 \mathrm{~cm}$ above the symphysis pubis for a length of about $10-12 \mathrm{~cm}$ was done. After the rectus fascia was opened, the rectus muscles were separated and dissected off the peritoneum, which was picked up between two tissue forceps and opened longitudinally. Dissection of the vesicouterine peritoneal fold to allow bladder retraction ('bladder flap'), that's in the bladder flap group. While in the non bladder flap group, uterine incision made 1 $\mathrm{cm}$ above the vesico-uterine reflection without incision and dissection of the bladder peritoneum.

The uterus was opened with a transverse lower segment cesarean section incision, and $20 \mathrm{IU}$ oxytocin (Oxytocin, Minapharm, Egypt) on dextrose 5\% infusion was given after the neonate had been delivered. After clamping of the umbilical cord, the women received $1.5 \mathrm{gm}$ Ampicillin-Sulbactam (Ultracillin, Sedico, Egypt) and $80 \mathrm{mg}$ garamycin (Epigent, EPICO, EGYPT).

The placenta was delivered using controlled cord traction techniques. Uterine incision repair was performed either extra abdominal or intra-abdominal (in-situ). The uterus was closed by absorbable continuous Vicryl 1 (Egysorb, Egypt) sutures in continuous double layer.

In all patients, rectus sheath closed by absorbable continuous Vicryl 2 (Egysorb, Egypt), Subcutaneous fat closed by absorbable continuous Vicryl 1and skin was closed by Vicryl 2-0 (Egysorb, Egypt) subcuticular sutures.

Postoperatively, all patients were given $75 \mathrm{mg}$ diclofenac sodium ampoule (Voltaren; Novartis, Cairo, Egypt) as intravenous infusion in dextrose $5 \%$ and $50 \mathrm{mg}$ diclofenac sodium tablets (Voltaren; Novartis, Cairo, Egypt) orally every 12 hours. All women received dextrose $5 \%$ was given by intravenous infusion every 8 hours for 24 hours post cesarean section. Blood loss was estimated using the weight difference method.

\subsection{Statistical Analysis}

Date entry and data analysis were done using SPSS version 19 (Statistical Package for Social Science). Data were presented as mean and standard deviation. Chi-square and Fisher Exact tests were used to compare between qualitative variables. Mann-Whitney test was used to compare between two quantitative variables in case of non-parametric data. $\mathrm{P}$-value considered statistically significant when $\mathrm{P}<0.05$.

\section{Results}

The study included 150 patients (75 in each arm). The baseline criteria were homogenous among both groups with no statistical significant differences (Table 1). The most common indication for CS in both groups was repeat CS $(41.2 \%)$ 
Table 1. Baseline criteria of the study participants.

\begin{tabular}{|c|c|c|c|}
\hline & $\begin{array}{l}\text { Bladder flap group } \\
(\mathrm{n}=75)\end{array}$ & $\begin{array}{l}\text { Non-bladder flap group } \\
(\mathrm{n}=75)\end{array}$ & $P$-value \\
\hline \multicolumn{4}{|l|}{ Maternal age: (years) } \\
\hline Mean \pm SD & $28.73 \pm 5.13$ & $28.00 \pm 6.08$ & 0.238 \\
\hline Range & $19.0-40.0$ & $19.0-42.0$ & \\
\hline \multicolumn{4}{|l|}{ Residence: No. (\%) } \\
\hline Rural & $5(6.7 \%)$ & $12(16.0 \%)$ & 0.071 \\
\hline Urban & $70(93.3 \%)$ & $63(84.0 \%)$ & \\
\hline \multicolumn{4}{|c|}{ Educational level: No. (\%) } \\
\hline Illiterate & $36(48.0 \%)$ & $28(37.3 \%)$ & \\
\hline Primary & $19(25.3 \%)$ & $17(22.7 \%)$ & 0.322 \\
\hline Preparatory & $15(20.0 \%)$ & $20(26.7 \%)$ & \\
\hline Secondary & $5(6.7 \%)$ & $10(13.3 \%)$ & \\
\hline \multicolumn{4}{|l|}{ BMI $\left(\mathrm{Kg} / \mathrm{m}^{2}\right)$} \\
\hline Mean \pm SD & $35.35 \pm 1.99$ & $34.94 \pm 1.94$ & 0.291 \\
\hline Range & $30.4-39.6$ & $29.3-39.1$ & \\
\hline \multicolumn{4}{|c|}{ Gestational age: (weeks) } \\
\hline Mean \pm SD & $37.27 \pm 2.52$ & $37.53 \pm 2.13$ & 0.517 \\
\hline Range & $32.0-41.0$ & $332.0-41.0$ & \\
\hline \multicolumn{4}{|l|}{ Parity: } \\
\hline Mean \pm SD & $2.16 \pm 1.59$ & $2.60 \pm 1.98$ & 0.364 \\
\hline Median (Range) & $2.0(0.0-8.0)$ & $2.0(0.0-9.0)$ & \\
\hline \multicolumn{4}{|l|}{ No. of CS: } \\
\hline Mean \pm SD & $1.57 \pm 1.18$ & $1.81 \pm 1.02$ & 0.245 \\
\hline Median (Range) & $1.0(0.0-4.0)$ & $0.0(0.0-3.0)$ & \\
\hline
\end{tabular}

BMI: body mass index, CS: cesarean section, SD: standard deviation.

Table 2 shows the intraoperative outcomes in both groups. There was aa significantly shorter skin incision to delivery time and total operative time in non-bladder flap group. Additionally, the mean estimated blood loss was significantly lower in the same group $(\mathrm{p}=0.000)$.

Table 3 shows the post operative outcomes in both groups. Postoperative pain score was significantly lower in the non-bladder flap group $(\mathrm{p}=0.000)$. No difference regarding the time of post operative defecation. No cases of postpartum hemorrhage, any postpartum complications or maternal mortality.

Table 4 shows that there was a statistical significant difference between the occurrences of microhematuria in both groups. Postoperative microheamturia was more common in the bladder flap group (133.87 \pm 104.39 vs. $24.13 \pm 68.22$, $\mathrm{p}=0.000)$. 
Table 2. The intraoperative outcomes of both study groups.

\begin{tabular}{|c|c|c|c|}
\hline Outcomes & $\begin{array}{l}\text { Bladder flap group } \\
\quad(\mathrm{n}=75)\end{array}$ & $\begin{array}{l}\text { Non-bladder flap group } \\
\qquad(\mathrm{n}=75)\end{array}$ & P-value \\
\hline \multicolumn{4}{|c|}{ Skin to delivery time (minutes): } \\
\hline Mean \pm SD & $10.79 \pm 2.96$ & $7.12 \pm 3.32$ & $0.000^{*}$ \\
\hline Range & $4.0-20.0$ & $2.0-15.0$ & \\
\hline \multicolumn{4}{|c|}{ Total operative time (minutes): } \\
\hline Mean \pm SD & $47.73 \pm 12.01$ & $40.51 \pm 10.53$ & $0.000^{*}$ \\
\hline Range & $17.0-90.0$ & $25.0-95.0$ & \\
\hline \multicolumn{4}{|c|}{ Estimated blood loss (ml): } \\
\hline Mean \pm SD & $526.00 \pm 79.42$ & $429.33 \pm 81.00$ & $0.000^{*}$ \\
\hline Range & $350.0-700.0$ & $300.0-700.0$ & \\
\hline
\end{tabular}

*Statistical significant difference.

Table 3. The postoperative outcomes of both study groups.

\begin{tabular}{|c|c|c|c|}
\hline & $\begin{array}{l}\text { Bladder flap group } \\
(\mathrm{n}=75)\end{array}$ & $\begin{array}{l}\text { Non-bladder flap } \\
\text { group }(n=75)\end{array}$ & P-value \\
\hline \multicolumn{4}{|c|}{ Postoperative pain score: } \\
\hline Mean \pm SD & $6.29 \pm 0.71$ & $5.81 \pm 0.90$ & $0.000^{*}$ \\
\hline Range & $4.0-7.0$ & $4.0-8.0$ & \\
\hline \multicolumn{4}{|c|}{$\begin{array}{l}\text { Postoperative defecation/passing: } \\
\text { (hours) }\end{array}$} \\
\hline Mean \pm SD & $10.89 \pm 1.46$ & $10.89 \pm 1.45$ & 0.758 \\
\hline Range & $6.0-15.0$ & $9.0-15.0$ & \\
\hline
\end{tabular}

*Statistical significant difference.

Table 4. The presence of preoperative and postoperative in the study groups.

\begin{tabular}{|c|c|c|c|}
\hline Microhematuria & $\begin{array}{l}\text { Bladder flap group } \\
(\mathrm{n}=75)\end{array}$ & $\begin{array}{l}\text { Non-bladder flap group } \\
\qquad(\mathrm{n}=75)\end{array}$ & P-value \\
\hline \multicolumn{4}{|l|}{ Pre-operative: } \\
\hline Mean \pm SD & $2.53 \pm 10.01$ & $4.60 \pm 29.46$ & 0.565 \\
\hline Median (Range) & $0.0(0.0-30.0)$ & $0.0(0.0-20.0)$ & \\
\hline \multicolumn{4}{|l|}{ Post-operative: } \\
\hline Mean \pm SD & $133.87 \pm 104.39$ & $24.13 \pm 68.22$ & $0.000^{*}$ \\
\hline Median (Range) & $50.0(0.0-250.0)$ & $0.0(0.0-200.0)$ & \\
\hline P-value ${ }^{2}$ & $0.000^{*}$ & $0.001^{*}$ & \\
\hline
\end{tabular}

*Statistical significant difference.

\section{Discussion}

Formation of the bladder flap is a standard step in traditional CS. It was mainly used in the pre-antibiotic era, as it was thought to decrease the rate of postoperative infection and allow better access for the lower uterine segment [7] [8]. Re- 
cently, it is believed the omission of the bladder flap in CS would have better outcomes with no risk.

Intraoperative results of our study showed an increase in both skin to delivery time and total operative time in group 1 compared to group 2 . The delay was 3 and 7 minutes respectively which was a statistically significant difference. Comparing the estimated blood loss demonstrated a statistically significant decrease in the non bladder flap compared to the bladder flap group. The estimated blood loss in group 1 and 2 was $526 \mathrm{cc}$ and $429.33 \mathrm{cc}$ respectively, which was a significant difference in favor of the non bladder flap group.

Postoperative values demonstrated better results in the non bladder flap technique compared with conventional bladder flap in terms of postoperative pain and microhematuria, which were both statistically significant.

In this study we conducted a different step that wasn't performed in previous researches, which was comparing the microhematuria pre- and post-operatively to rule out any bias, and the results showed a marked significance post-operatively showing mean $133.87 \pm 104.39$ in bladder flap group and 24.13 \pm 68.22 in non bladder flap group, which concludes that even though there was no bladder injury but in the intraoperative complications in both group, nonetheless it does mean there is micro injury on the tissues level when dissecting.

Yet, the postpartum passing, defecation and hemorrhage and the postoperative complications showed no difference between the two groups.

Previous studies have been discussing the omission of bladder flap step in CS. In 2001, Hohlagschwandtner et al. in Vienna conducted the study on 102 women which were divided into two groups; results showed better outcomes on the level of total operative time, skin to delivery time, median blood loss, microhematuria and median need for analgesia but showed no difference in bowel function. This study's results were similar to our study results expect that it was conducted on women with no scarred uterus [9].

Another study was conducted exclusively on women with primary CS by Chigbu et al. [8]. A 3-year, prospective, non-randomized study of 142 primary cesarean sections with omission of bladder flap formation was carried out. Comparing this study to previous one and our study, the observed mean estimated blood loss was similar. The mean hemoglobin concentration difference supported blood loss estimations. The mean duration of surgery was slightly higher than that of the "omission of bladder flap" in our study and the previous study.

One of the studies carried out on women with both primary and repeated CS was St. Louis school of Medicine in 2012 where 258 women were randomized into creation or omission of bladder flap. The median skin incision to delivery interval was shorter with omission of the bladder flap (9 [range 1 - 43] compared with 10 [range 2 - 70] minutes), but there was no difference in total operating time (51 [range 18 - 124] minutes compared with 51 [range 16 - 178]). No bladder injuries occurred in either group and there were no significant differences in estimated blood loss, change in hemoglobin level, postoperative microhematu- 
ria, postoperative pain, hospital days, endometritis, or urinary tract infection. [10].

Another study from Egypt also was conducted by Elsersy, including 1674 women with primary and repeated CS in Alexandria University divided into two groups of non-bladder flap and bladder flap. Her results showed that the total operating time in the non-bladder flap group ranges from 25 to 140 minutes with median of $40 \pm 11.4$ minutes, which is significantly shorter than that of the bladder flap group. Also the skin incision to delivery time in the non bladder flap group is ( $5 \pm 1.3 \mathrm{~min}$ range $1-20 \mathrm{~min}$ ) which is significantly shorter. Analysis of data shows significantly less microhematuria. There is significant decrease in $\mathrm{Hb}$ level range post-operative in the bladder flap group (1.1 \pm 0.6 range 0.6 $2.1 \mathrm{gm} / \mathrm{dl})$. This study had strikingly similar results to our study especially in terms of skin to delivery and total operation time [11].

An important point that must be mentioned is the absence of bladder injury in both groups in our study and all the mentioned studies. This can be explained by the fact that the incidence of bladder flap during C.S so low $(0.14 \%-0.35 \%)$, a sample size of 40,000 women would have been needed to be accurately assessed. This is why we compared the presence of microhematuria in our study both pre and postoperatively to assess any micro injuries in the two groups.

The results match the Pelosi technique which introduced non bladder flap in the first place [12] and previous researches on the subject [9] [11]. We find that the covenantal dissection of the bladder flap routinely done in traditional CS is not necessary and can be safely omitted unless it's indicated in highly adhesive fields as in repeat CS. Herein, the vesico-uterine segment (top of bladder) is sometimes thickened and thus one has sometimes difficulty in "definitely determine" the bladder top. If not-carefully, one may misunderstand the fold and may injure the bladder

Although we believe that a study on the long term effects should be conducted, yet considering our results we believe that omitting the bladder flap in standard technique of CS is not only safe but can even have more advantages over the classic dissection of bladder flap.

\section{Conflicts of Interest}

The authors declare that they have no conflict of interest.

\section{References}

[1] Stokke, S.J. and Becher, L.M.W. (2013) Indications for Cesarean Section at St. Joseph Medical Hospital, Moshi Tanzania.

[2] Belizán, J.M., et al. (1999) Rates and Implications of Caesarean Sections in Latin America: Ecological Study. BMJ, 319, 1397-1402.

https://doi.org/10.1136/bmj.319.7222.1397

[3] Betrán, A.P., et al. (2016) The Increasing Trend in Caesarean Section Rates: Global, Regional and National Estimates: 1990-2014. PLOS ONE, 11, e0148343. https://doi.org/10.1371/journal.pone.0148343 
[4] Wood, R.M., Simon, H. and Oz, A.U. (1999) Pelosi-Type vs. Traditional Cesarean Delivery. A Prospective Comparison. The Journal of Reproductive Medicine, 44, 788-795.

[5] Berghella, V., Baxter, J.K. and Chauhan, S.P. (2005) Evidence-Based Surgery for Cesarean Delivery. American Journal of Obstetrics \& Gynecology, 193, 1607-1617. https://doi.org/10.1016/j.ajog.2005.03.063

[6] Walsh, C.A. (2010) Evidence-Based Cesarean Technique. Current Opinion Obstetrics and Gynecology, 22, 110-115. https://doi.org/10.1097/GCO.0b013e3283372327

[7] Malvasi, A., et al. (2010) Should the Visceral Peritoneum at the Bladder Flap Closed at Cesarean Sections? A Post-Partum Sonographic and Clinical Assessment. The Journal of Maternal-Fetal \& Neonatal Medicine, 23, 662-669. https://doi.org/10.3109/14767050903358363

[8] Chigbu, C.O., Ezeome, I.V. and Iloabachie, G.C. (2006) Non-Formation of Bladder Flap at Cesarean Section. International Journal of Gynecology \& Obstetrics, 95, 284-285. https://doi.org/10.1016/j.ijgo.2006.08.010

[9] Hohlagschwandtner, M., et al. (2001) Is the Formation of a Bladder Flap at Cesarean Necessary? A Randomized Trial. Obstetrics \& Gynecology, 98, 1089-1092. https://doi.org/10.1016/S0029-7844(01)01570-8

[10] Tuuli, M.G., et al. (2012) Utility of the Bladder Flap at Cesarean Delivery: A Randomized Controlled Trial. Obstetrics \& Gynecology, 119, 815-821. https://doi.org/10.1097/AOG.0b013e31824c0e12

[11] Elsersy, M.A. (2016) Usefulness of Bladder Dissection in Cesarean Section: A Randomized Controlled Trial. International Journal of Reproduction, Contraception, Obstetrics and Gynecology, 5, 4317-4320. https://doi.org/10.18203/2320-1770.ijrcog20164335

[12] Pelosi, M.A. and Ortega, I. (1994) Cesarean Section: Pelosi's Simplified Technique. Revista Chilena de Obstetricia y Ginecologia, 59, 372-377. 